\title{
Lentiviral vector-mediated siRNA knockdown of the YAP gene inhibits growth and induces apoptosis in the SGC7901 gastric cancer cell line
}

\author{
ZHOU ZHOU, JIN-SHUI ZHU, ZHI-PENG XU and QIANG ZHANG \\ Department of Gastroenterology, Shanghai Sixth People's Hospital Affiliated to \\ Shanghai Jiaotong University, Shanghai 200233, P.R. China
}

Received March 23, 2011; Accepted July 22, 2011

DOI: $10.3892 / \mathrm{mmr} .2011 .543$

\begin{abstract}
Gastric carcinoma is among the most prevalent malignancies, and a leading cause of cancer deaths worldwide. The Hippo pathway defines a novel signaling pathway regulating cell proliferation. The key factor in this kinase cascade is the transcriptional co-activator yes-associated protein (YAP), which is constitutively activated in various types of cancer, including gastric carcinoma. To determine the effect on SGC7901 gastric cancer cells after inhibition of the YAP expression, we used lentivirus-derived small hairpin RNA (shRNA) against YAP. The YAP-specific shRNA significantly suppressed YAP expression at the mRNA and protein levels, reduced cancer cell proliferation and promoted cancer cell apoptosis. It was also found that YAP knockdown significantly increased the G0/G1 cell population and reduced expression levels of a number of genes crucial to cell proliferation and apoptosis, including Ki-67, proliferating cell nuclear antigen (PCNA), survivin and cyclin D1. Thus, our data suggested that knockdown of YAP expression plays a significant role in gastric cancer cell proliferation and apoptosis. Therefore, the YAP gene serves as a potential therapeutic target in gastric cancers.
\end{abstract}

\section{Introduction}

Gastric carcinoma is one of the most common malignant types of cancer worldwide, with characteristics such as high incidence, ease of transfection and a high mortality rate. According to statistics, the global number of newly diagnosed gastric cancer patients is approximately 800,000 per year. Gatric cancer accounts for $9 \%$ of newly diagnosed cancer patients, and ranks fourth after lung, breast and colon cancer (1). Gastric

Correspondence to: Dr Jin-Shui Zhu, Department of Gastroenterology, Sixth People's Hospital Affiliated to Shanghai Jiaotong University, Shanghai 200233, P.R. China

E-mail: zhujs1803@hotmail.com

Key words: YAP gene, RNA interference, cell apoptosis, cell proliferation cancer incidence is particularly high in Asia. According to a retrospective study, early gastric cancer only accounts for $2-4 \%$, whereas most cases tend to be advanced gastric cancer (1). At present, the main treatment available for advanced gastric cancer are surgery and chemotherapy. However, such treatments are not efficacious; thus, new methods of treatment for advanced gastric carcinoma should be found.

YAP, a $65 \mathrm{kDa}$ proline-rich phosphoprotein, located at locus 11q22, was originally identified due to its interaction with the SH3 domain of the c-Yes proto-oncogene product (2). Several experiments have confirmed that YAP exhibits oncogenic activities. In the human mammary epithelial cell line MCF10, overexpression of YAP induces epithelial-to-mesenchymal transition, and suppression of apoptosis, promoting anchorage independent growth in soft agar, suggesting that YAP contributes to malignant transformation in cancer (3). YAP has been shown to cooperate with cIAP1 to stimulate tumor growth in nude mice. Moreover, transgenic mice with liver-specific YAP overexpression showed a marked increase in liver size and eventually developed tumors (4). A strong and diffuse nuclear and cytoplasmic YAP expression in colonic adenocarcinoma, lung adenocarcinoma, and ovarian serous cystadenocarcinoma have also been observed (5). The abovementioned data strongly indicate the role of YAP as an oncogene.

RNA interference (RNAi) has emerged as the most effective gene silencing tool. In recent years, shRNA, which produces a tight hairpin turn, has been used to induce RNAi in mammalian cells, whereby a vector containing shRNA fragment is introduced into a cell and transcribed by RNA polymerase III. The resulting shRNA hairpin structure is processed by the cell mechanisms to generate 21-25 nt of small interfering RNA (siRNA). siRNA mediates degradation of its complementary mRNAs (6). This technology has numerous applications in biomedical research, and health care studies such as HIV, viral hepatitis, cardiovascular, cerebrovascular diseases, metabolic diseases, neurodegenerative disorders and cancers (7).

Studies have found YAP expression to be significantly high in gastric carcinoma as compared to normal gastric epithelium and gastric intestinal metaplasia epithelium (8). However, its role in the pathological process of gastric carcinoma has yet to be clarified. Moreover, biological effects from the inhibition 
of YAP expression have yet to be determined. In our study, RNAi technology was used to investigate the effects on cell proliferation, apoptosis and cell cycle alteration after YAP gene silencing in vitro using the human gastric cancer cell line SGC7901.

\section{Materials and methods}

Materials. 3-(4,5)-Dimethylthiahiazo(-z-yl)-3,5-diphenytetrazoliumromide (MTT) was from Dingguo Biology (Shanghai, China); Dulbecco's modified Eagle's medium (DMEM) and fetal bovine serum (FBS) were obtained from Thermo Fisher Scientific Inc. (Waltham, MA, USA). Cell lines used in the experiment (SGC7901 cells and 293T cells) were obtained from the Institute of Biochemistry and Cell Biology (Shanghai); TRIzol reagent and Lipofectamine 2000 were obtained from Invitrogen (Carlsbad, CA, USA); M-MLV reverse transcriptase was obtained from Promega (Madison, WI, USA); SYBR-Green master mixture was obtained from Takara (Otsu, Japan); PGC-LV vector and virion-packaging elements (pHelper 1.0 and pHelper 2.0) were obtained from Genechem (Shanghai); all antibodies were obtained from Santa Cruz Biotechnology (Santa Cruz, CA, USA); the cell cycle analysis and apoptosis kits [propidium iodide (PI), RNase A, Annexin V-FITC] were obtained from KeyGEN Biology (Nanjing, China), and the ECL-PLUS/Kit was obtained from GE Healthcare (Piscataway, NJ, USA).

Cell culture. SGC7901 cells and 293T cells were cultured in DMEM supplemented with $10 \%$ heat-inactivated FBS, $100 \mathrm{U} /$ $\mathrm{ml}$ of penicillin and $100 \mu \mathrm{g} / \mathrm{ml}$ of streptomycin. The cells were placed in a humidified atmosphere containing $5 \% \mathrm{CO}_{2}$ at $37^{\circ} \mathrm{C}$.

Preparation of lentivirus YAP shRNA. The sequences of siRNA duplexes targeting the YAP gene (NM_001130145) were designed online (http://rnaidesigner.invitrogen.com/ rnaiexpress/rnaiExpress.jsp). The sequences were designed as follows: YAP1 (5'-GGTCCTCTTCCTGATGGAT-3'), YAP2 (5'-CTCAGGATGGAGAAATTTA-3'), YAP3 (5'-CAG GTGATACTATCAACCAAA-3') and YAP4 (5'-GAC CAATAGCTCAGATCCTTT-3'). The hairpin DNA oligomers were synthesized and annealed. The annealed double-stranded shRNA oligos were cloned into a PGC-LV neo lentivirus vector that was driven by the U6 promoter and carried the transgene for green fluorescent protein. A control shRNA unrelated to human gene sequences was used as a negative control (5'-TTCTCCGAACGTGTCACGT-3') (9). The accuracy of the inserted sequences of the vectors were verified by sequencing. We selected the most efficient recombinant vector from YAP1, YAP2, YAP3 and YAP4 by transfecting SGC7901 cells and detecting their inhibition level using Western blotting. The selected vector was denoted as YAP-shRNA. YAP-shRNA and NC vector were then placed into 293T cells with virion-packaging elements (pHelper 1.0 and pHelper 2.0). YAP-shRNA and NC vector were separately cotransfected into 293T cells with packing plasmids by calcium phosphate precipitation. After a $24 \mathrm{~h}$ culture, the viral supernatant of each clone was collected and the virus titer was measured according to standard protocol.
Selection of the cells in which lentivirus vectors have a stable expression. Cells were washed and switched into complete medium after transfection. Stable cell lines containing YAPshRNA or NC vector were selected, and $25 \mu \mathrm{g} / \mathrm{ml}$ of puromycin were added into the medium. After 6 weeks of culturing in the presence of puromycin, the remaining cells were isolated and transferred into 24-well dishes. One section of the selected clones was removed for the subsequent experiments and the remaining sections were frozen for future use.

$q R T-P C R$. To quantitatively determine the mRNA expression level, the qRT-PCR method was used. The total RNA of each clone was extracted using TRIzol reagent according to the manufacturer's protocol. Reverse-transcription was carried out using M-MLV and cDNA amplification was carried out using the SYBR-Green master mix kit according to the manufacturer's protocol. YAP, Ki-67, survivin, AFP, PCNA, cyclinA, cyclinD1 and cyclinE genes were amplified using specific oligonucleotide primers, and the human glyceraldehyde3-phosphate dehydrogenase (GAPDH) gene was used as an endogenous control. The sequences of the primers and size of the products are shown in Table I. Data were analyzed using the comparative $\mathrm{Ct}$ method $\left(2^{-\Delta \Delta \mathrm{Ct}}\right)(10)$. Three separate experiments were performed for each clone.

Western blot analysis. Cells of each group were harvested and extracted using lysis buffer (Tris-HCl, SDS, mercaptoethanol and glycerol). Cell extracts were boiled for $5 \mathrm{~min}$ in loading buffer and an equal amount of cell extracts was separated on $15 \%$ SDS-PAGE gels. Separated protein bands were transferred into polyvinylidene fluoride (PVDF) membranes and the membranes were blocked in 5\% skimmed milk powder. The primary antibodies against YAP, Ki-67, survivin, AFP, PCNA, cyclinA, cyclinD1 and cyclinE genes were diluted according to the manufacturer's instructions, and incubated overnight at $4^{\circ} \mathrm{C}$. Horseradish peroxidase-linked secondary antibodies were then added at a dilution ratio of 1:1000, and incubated at room temperature for $2 \mathrm{~h}$. Finally, the membranes were washed with PBS three times and the immunoreactive bands were visualized using an ECL-PLUS/Kit according to the manufacturer's instructions. The relative protein level in different cell lines was normalized to the GAPDH concentration. Three separate experiments were performed for each clone.

Cell proliferation assay. Cell proliferation was analyzed with the MTT assay. Cells were incubated in 96-well-plates at a density of $1 \times 10^{5}$ cells/well with DEME supplemented with $10 \%$ FBS. Cells were treated with $20 \mu 1$ MTT dye at 24, 48, and $72 \mathrm{~h}$ and then incubated with $150 \mu 1$ of DMSO for $5 \mathrm{~min}$. The color reaction was measured at $570 \mathrm{~nm}$ using an enzyme immunoassay analyzer (model680, Bio-Rad,Hercules, CA, USA). The proliferation rate was calculated for each clone. The mean and standard deviation of five relative proliferation rates for each clone were calculated.

Cell cycle analysis. To detect cell cycle variation, cells were trypsinized, washed with PBS and fixed with $80 \%$ cold ethanol overnight at $-20^{\circ} \mathrm{C}$. After PBS washing, the fixed cells were stained with PI in the presence of RNase/A for 30 min at room temperature in the dark. Each sample was filtered through 
Table I. Primer sequences.

\begin{tabular}{|c|c|c|}
\hline Primers & Sequences & Size of products (bp) \\
\hline YAP (forward) & CCTGATGGATGGGAACAAGC & 134 \\
\hline YAP (reverse) & GCACTCTGACTGATTCTCTGG & \\
\hline Ki-67 (forward) & GGAACAGCCTCAACCATCAG & 210 \\
\hline Ki-67 (reverse) & ССАСТСТТТСТСССТССТСТС & \\
\hline Survivin (forward) & GGACCACCGCATCTCTACA & 338 \\
\hline Survivin (reverse) & GCACTTTCTTCGCAGTTTCC & \\
\hline AFP (forward) & ACCATGAAACAAGAGCTTCTCA & 164 \\
\hline AFP (reverse) & GAAATCAACTTTGGACCCTCT & \\
\hline PCNA (forward) & TGTCCGTCCAAGCAGAGG & 107 \\
\hline PCNA (reverse) & CGCACAAGAGTTCCGTAGC & \\
\hline CyclinA (forward) & CCCAGAAGTAGCAGAGTTTGTG & 295 \\
\hline CyclinA (reverse) & TTGTCCCGTGACTGTGTAGAG & \\
\hline CyclinD1 (forward) & GGTGGCAAGAGTGTGGAG & 148 \\
\hline CyclinD1 (reverse) & CCTGGAAGTCAACGGTAGC & \\
\hline CyclinE (forward) & AGGTTTCAGGGTATCAGTGGTGC & 189 \\
\hline CyclinE (reverse) & СTTTCTTTGCTCGGGCTTTGTCC & \\
\hline GAPDH (forward) & TGACTTCAACAGCGACACCCA & 121 \\
\hline GAPDH (reverse) & CACCCTGTTGCTGTAGCCAAA & \\
\hline
\end{tabular}

a $50-\mu \mathrm{m}$ nylon filter to obtain single-cell suspension. The samples were then analyzed on a FACsort flow cytometer (Becton-Dickinson, Mountain View, CA, USA). ModFit3.0 software (Verity Software House, Topsham, ME, USA) was used for the cell cycle analysis. Three separate experiments were performed for each clone.

Cell apoptosis analysis. To detect cell apoptosis, cells were trypsinized, washed with cold PBS and resuspended in binding buffer according to the instructions of the apoptosis kit. FITCAnnexinV and PI were added to the fixed cells for $20 \mathrm{~min}$ in the dark at room temperature. Annexin V-binding buffer was then added to the mixture before fluorescence was measured on a FACsort flow cytometer. Cell apoptosis was analyzed using CellQuest software (Becton-Dickinson). Three separate experiments were performed for each clone.

Statistical analysis. The result of each experiment was shown as the mean \pm SD where applicable. Statistically significant differences in each assay was determined by SPSS, version 15.0. The difference in each group were tested for significance using one-way analysis of variance (ANOVA). $\mathrm{P}<0.05$ was considered to be statistically significant.

\section{Results}

Construction and selection of the most effective YAP-specific shRNA expression vector. The sequencing results showed that the shRNA coding sequences in recombinant plasmids corresponded exactly with our designed target nucleic acid sequences. The construction of recombinant plasmids YAP1, YAP2, YAP3 and YAP4 was found to be successful (Fig. 1A).
The inhibition levels of the four sets were detected by Western blotting $48 \mathrm{~h}$ after transfection. The results showed that the transfection of YAP1, YAP2, YAP3 and YAP4 inhibited the YAP expression in SGC7901 cells. In particular, the most obvious gene-silencing effect was observed in YAP2, which reduced the YAP gene expression by $87.1 \%$ (Fig. 1B). Therefore, YAP2 was selected for stable transfection and its virus titer was $3 \times 10^{8} \mathrm{TU} / \mathrm{ml}$. After selecting the cells with stable transfection, they were observed under a fluorescence microscope system (Olympus micropublisher 3.3RTV, Center Valley, PA, USA) to obtain green fluorescence (Fig. 1C) and we found that there was no alteration in cellular morphology after transfection.

In this study, the clone in which the YAP-shRNA virus vectors transfected was known as the YAP-shRNA group, the negative control vectors transfected were known as NC group and no vector transfected was known as the CON group.

Silencing of YAP expression by stable transfection of $Y A P$-shRNA. The SGC7901 cells were shown to exhibit a high YAP gene expression in preliminary experiments (data not shown). To examine the effect of YAP-shRNA treatment on the SGC7901 cell line, the YAP mRNA expression level was measured by qRT-PCR. As shown in Fig. 2A, an obvious inhibition of YAP mRNA expression was observed in the YAP-shRNA group compared with the NC and CON groups $(0.16 \pm 0.01$ vs. $1.01 \pm 0.03, \mathrm{P}<0.05 ; 0.16 \pm 0.01$ vs. $1.10 \pm 0.09$, $\mathrm{P}<0.05$, respectively). As for the inhibition of the protein level, an obvious inhibition of the YAP protein expression was observed in the YAP-shRNA group compared with the other two groups after RNAi $(0.26 \pm 0.01$ vs. $0.89 \pm 0.05, \mathrm{P}<0.05$; $0.26 \pm 0.01$ vs. $0.86 \pm 0.02, \mathrm{P}<0.05$, respectively) (Fig. $2 \mathrm{~B}$ ). 
A

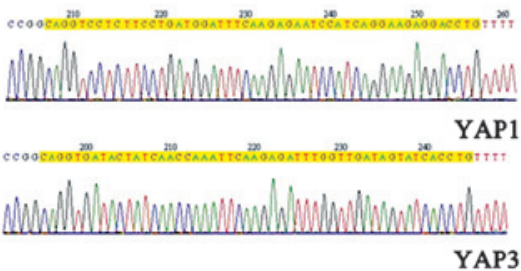

B
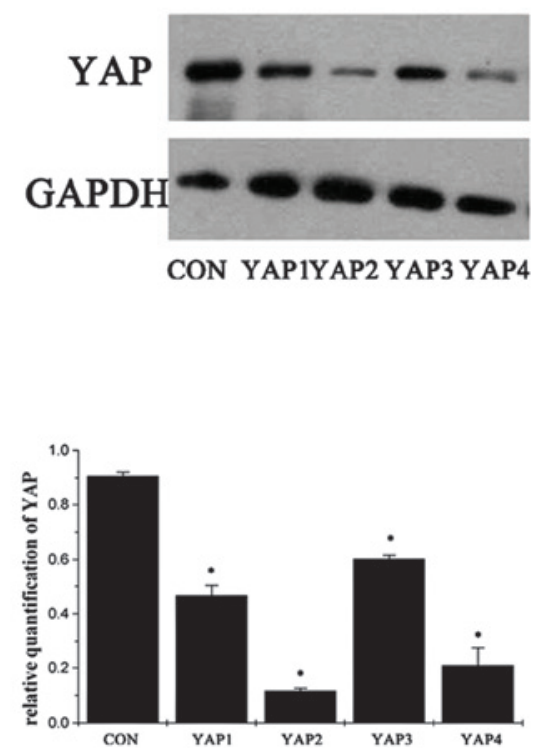

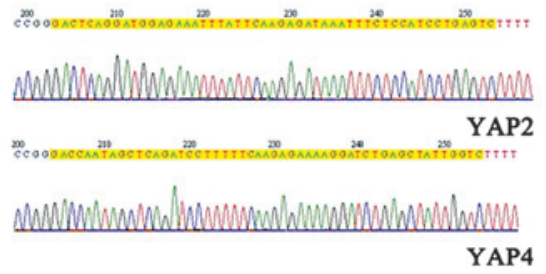

C
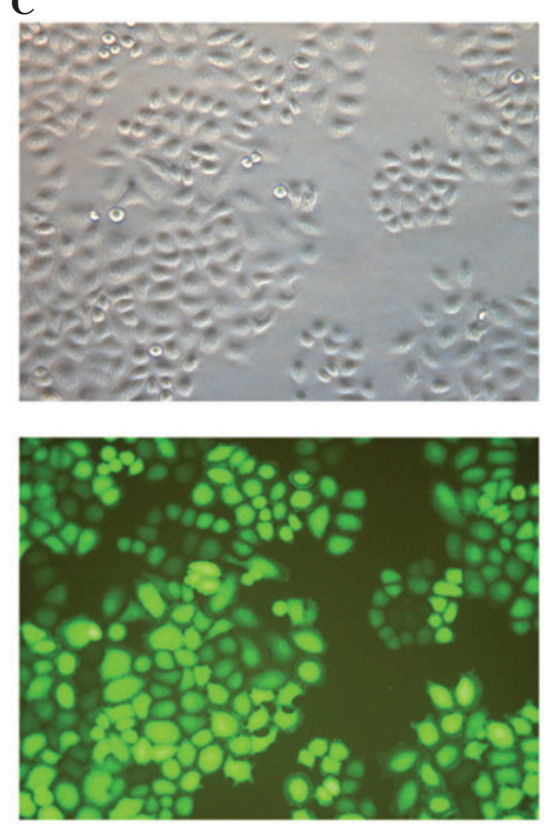

Figure 1. The sequencing results of recombinant sets and their inhibition level in SGC7901 cells. (A) The sequencing results of YAP1, YAP2, YAP3 and YAP4. (B) Total cellular proteins were extracted after transfection and determined by Western blot analysis using antibodies against YAP. GAPDH was used as an endogenous control. Data are the mean \pm SD of three independent experiments. $\mathrm{P}<0.05$, compared with CON group. Western blotting gel result (upper panel); bar graph (lower panel). (C) After stable transfection with hydromycin, the cells were observed under a fluorescence microscope system. Light micrograph (magnification, x400) (upper panel); fluorescent micrograph (magnification, x400) (lower panel).

A

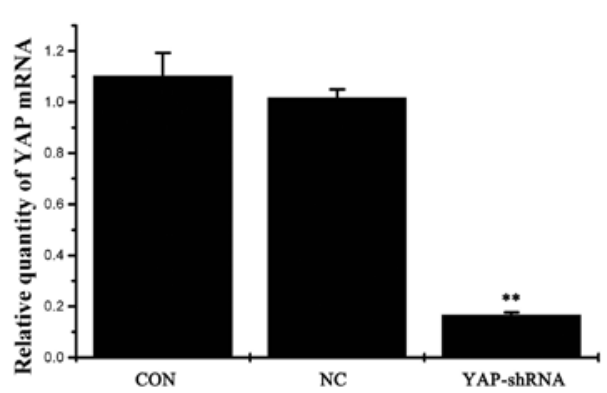

C

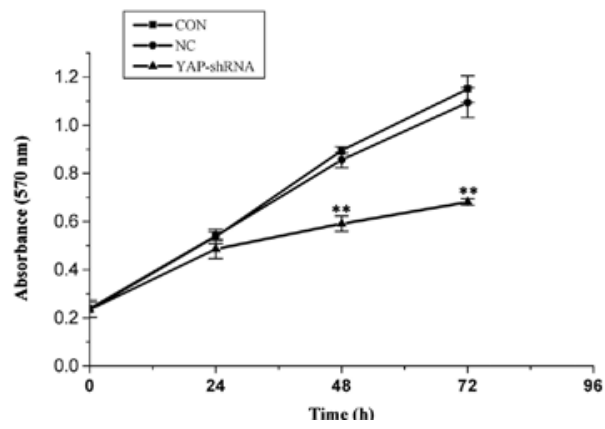

B

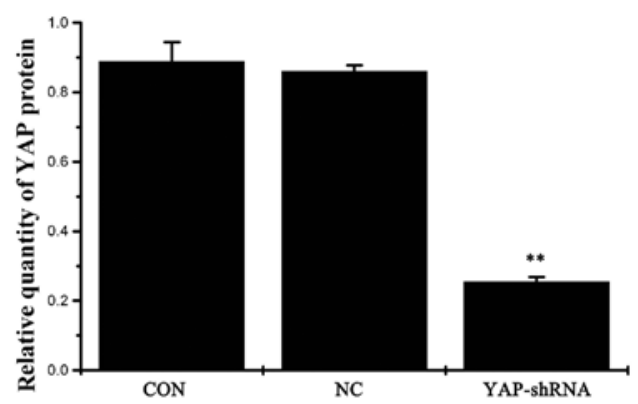

YAP

GAPDH

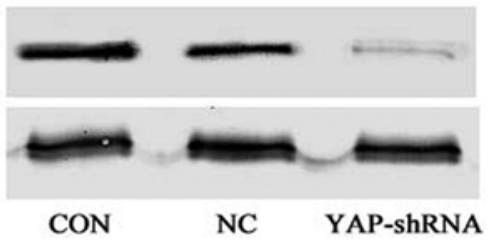

Figure 2. YAP-shRNA stably decreased YAP expression and caused decreased cell proliferation in SGC7901 cells. (A) YAP mRNAs were detected by qRT-PCR after RNAi treatment. GAPDH was used as an endogenous control. ${ }^{* *} \mathrm{P}<0.01$, compared with the NC group and CON group. (B) YAP proteins were detected by Western blotting after RNAi treatment. GAPDH was used as an endogenous control. ${ }^{* *} \mathrm{P}<0.01$, compared with the NC and CON groups. Bar graph (upper panel); Western blotting gel result (lower panel). (C) The proliferation curves of SGC7901 cells during 3 days evaluated by MTT assay. 
A

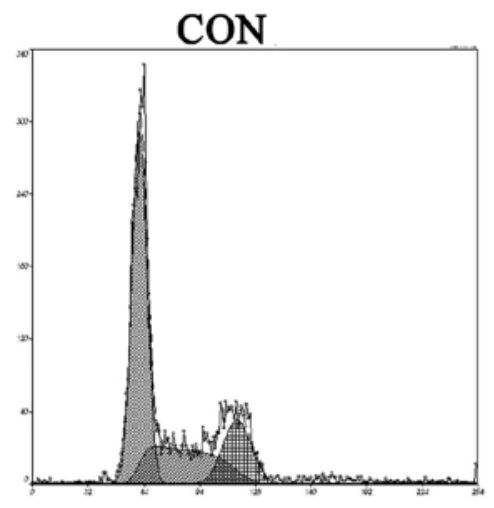

B

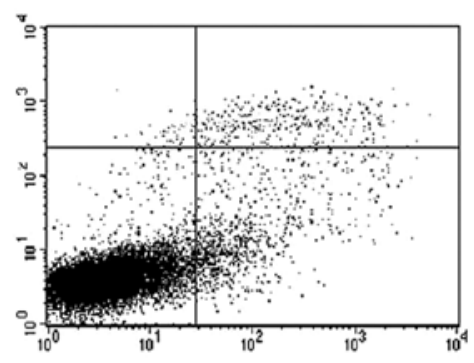

NC
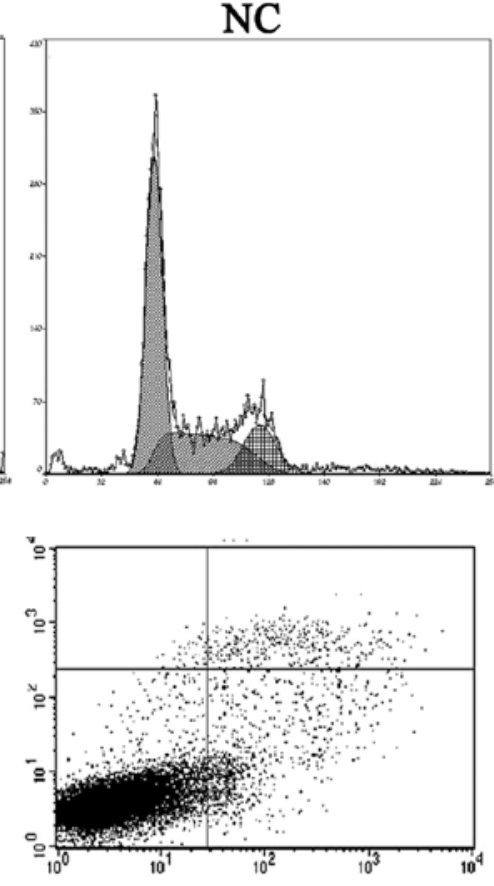

D $\quad \mathrm{Ki}-67 \stackrel{\text { CON }}{=} \underset{\mathrm{NC}}{\longrightarrow} \stackrel{\text { YAP-shRNA }}{\longrightarrow}$
C

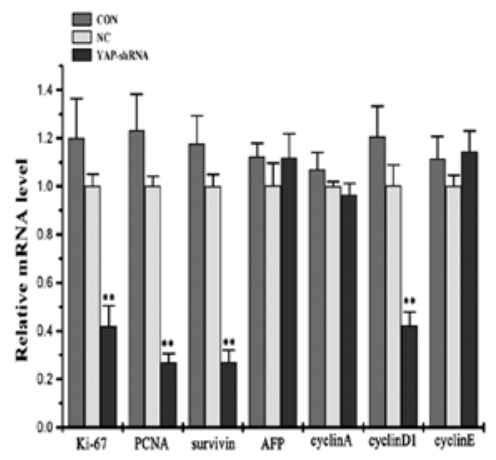

Survivin
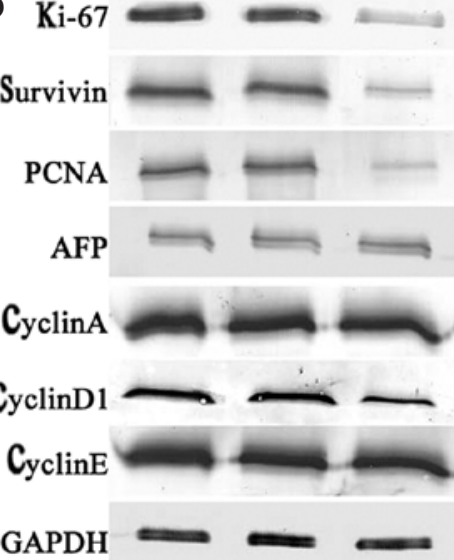
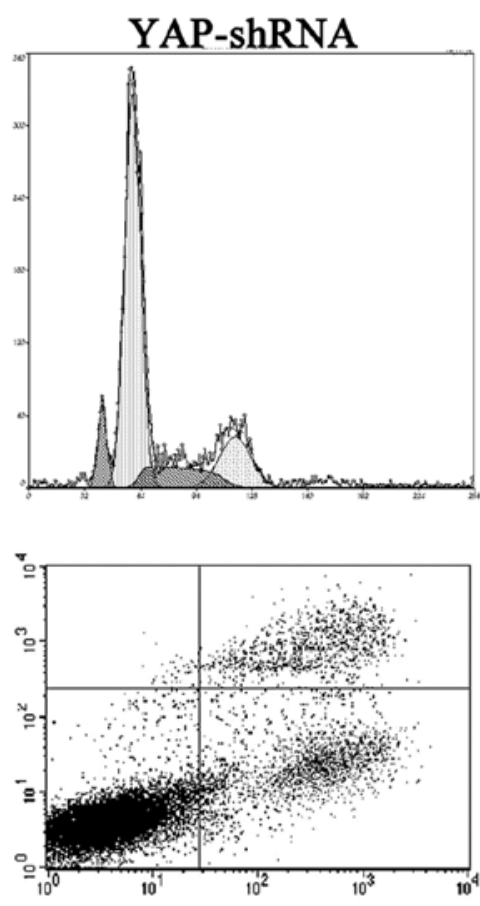$$
\text { (1) }
$$

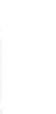

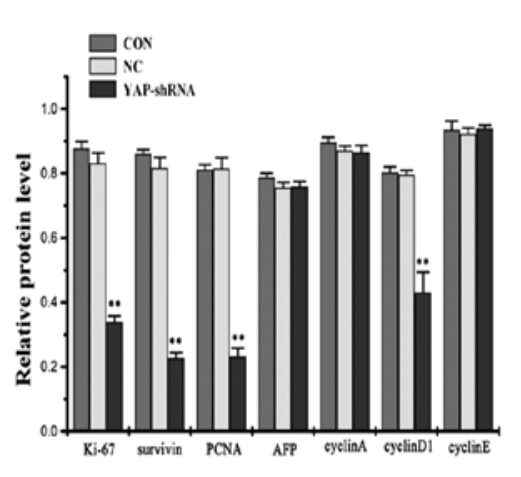

Figure 3. Effect of down-regulation of YAP expression on cell cycle, cell apoptosis and cytokines in SGC7901 cells. (A) Cell cycle was analyzed after RNAi treatment. Knockdown of YAP expression induced G1 arrest in SGC7901 cells. (B) Cell apoptosis was analyzed after RNAi treatment. Knockdown of YAP expression promoted apoptosis in SGC7901 cells. (C) The mRNA level of Ki-67, PCNA, survivin, AFP, cyclin A, cyclin D1 and cyclin E were detected by qRT-PCR after RNAi treatment. GAPDH was used as an endogenous control. ${ }^{* *} \mathrm{P}<0.01$, compared with the NC and CON groups. (D) The protein levels of Ki-67, PCNA, survivin, AFP, cyclin A, cyclin D1 and cyclin E were detected by Western blotting after RNAi treatment. GAPDH was used as an endogenous control. ${ }^{* *} \mathrm{P}<0.01$, compared with the $\mathrm{NC}$ and $\mathrm{CON}$ groups. Western blotting gel result (left panel); bar graph (right panel).

YAP siRNA inhibited cell growth. Deregulated cell proliferation is a hallmark of cancer (11). To determine whether the silencing of YAP by RNAi had an inhibitory effect on SGC7901 cell proliferation, we carried out MTT assay. As shown in Fig. 2C, compared with the NC and CON groups, the YAP-shRNA group was associated with an obvious inhibition in cell proliferation, wheras no obvious difference was observed in cell proliferation in the $\mathrm{NC}$ and CON groups. These data suggest that YAP played a critical role in gastric cancer cell proliferation.

Down-regulation of YAP induced G0/G1 arrest. To determine the effect of YAP suppression on the SGC7901 cell cycle, flow cytometry with PI staining was performed. The proportion of cells in the G0/G1 phase in the YAP-shRNA group was significantly increased compared with that of the NC and CON groups. As shown in Fig. 3A, 74.17\% cells of the YAP-shRNA group accumulated in the G0/G1 phase in contrast with 52.3 and $54.4 \%$ of the NC and CON groups, respectively. The S-phase fraction in the CON and NC groups was 26.9 and $31.9 \%$, respectively, but in the YAP-shRNA group the value decreased to $8.73 \%$. No significant differences were observed in the proportion of $\mathrm{G} 2 / \mathrm{M}$ fractions among the three groups, and an apoptotic peak was detected in the YAP-shRNA group, indicating that apoptosis occurred after the YAP gene was silenced. These results suggested that in vitro conditions and siRNA-mediated down-regulation of the YAP gene induced obvious cell proliferation inhibition. 
Down-regulation of YAP induced cell apoptosis. To determine the effect of YAP suppression on SGC7901 cell apoptosis, flow cytometry with PI/FITC-AnnexinV staining was performed. The results showed that the apoptotic rate of cells treated with YAP shRNA vector (42.1\%) was significantly higher than that of the CON $(9.75 \%)$ and $\mathrm{NC}(10.69 \%)$ groups (Fig. 3B). Findings also showed that knockdown of YAP expression by RNAi induced apoptosis of SGC7901 cells.

YAP silencing reduced Ki-67, PCNA, survivin and cyclinD1 expression. We have shown that YAP accelerated cell proliferation and inhibited cell apoptosis in gastric cancer cells. To further explore the molecular mechanisms of the role of YAP, we focused on various recognized proliferation- and apoptosis-associated genes. qRT-PCR and Western blotting were performed to determine the Ki-67, PCNA, survivin and AFP levels after silencing YAP by RNAi in SGC7901 cells. As shown in Fig. 3C and D, knockdown of YAP led to downregulation of the expression of $\mathrm{Ki}-67, \mathrm{PCNA}$ and survivin mRNA and protein compared to the CON $(\mathrm{P}<0.01)$ and NC $(\mathrm{P}<0.01)$ groups. However, no significant difference was observed on the AFP expression regardless of whether or not the YAP expression was suppressed ( $>0.05$ ), indicating that YAP enhanced proliferation and induced apoptosis in gastric cancer cells via mechanisms involving Ki-67, PCNA and survivin.

YAP silencing induced G1 phase arrest in the above experiments. Since cyclins play a crucial role in cell progression, the mRNA and protein levels of cell cycle regulatory factors including cyclinD1, cyclinE and cyclinA were examined. The mRNA and protein levels of cyclinD1, whose implementation was crucial for the G1 to S transition (12), were significantly down-regulated $(\mathrm{P}<0.01)$, whereas no significant variations were observed in cyclin $\mathrm{A}(\mathrm{P}>0.05)$ and cyclinE $(\mathrm{P}>0.05)$ (Fig. 3C and D). This observation coincided with the result of the cell cycle alteration, where the G1 phase was obviously delayed in the YAP-shRNA group compared with the other two groups.

\section{Discussion}

The ability to control tissue homeostasis is key to all multicellular organisms. Dysregulation of either proliferation or apoptosis may lead to cell malignant transformation (13). Studies in Drosophila melanogaster have shown the Hippo signaling pathway, which acts as a potent regulator of inhibition of cell growth and promotion of cell apoptosis. This pathway is defined by a kinase cascade whereby Mst1/2, facilitated by WW45, phosphorylates and activates Lats $1 / 2$. Lats1/2 phosphorylates and inactivates YAP. When YAP is phosphorylated by the Lats $1 / 2$, it reserves in the cytoplasm, whereas it interacts with 14-3-3 proteins and is thought to be inactive. Constitutive activation of YAP promotes the downstream factor transcription and expression, which induces cell proliferation, inhibits cell apoptosis, overcomes contact inhibition and contributes to tumorigenesis. Therefore, Hippo signaling antagonizes YAP function by changing its subcellular localization, loss of Hippo signaling promotes nuclear accumulation of YAP and consequently induces the downstream factor activation $(14,15)$.
Evidence suggests that YAP is the most critical effector in the Hippo pathway and its overexpression leads to oncogenesis (16). We found that down-regulation of YAP expression by siRNA inhibited cell proliferation. Furthermore, cell cycle analysis showed that the percentage of the G0/G1 phase cells was significantly elevated in the YAP-shRNA group compared with that in the $\mathrm{NC}$ and $\mathrm{CON}$ groups, indicating a G0/G1 phase arrest of the cell cycle. Cell proliferation is regulated by cell cycle mechanisms and depends on the balance between positive factors (cyclins) and negative factors (cyclindependent kinase inhibitors) (12). In the present study, we detected the expression of cyclin A, cyclinD1 and cyclinE. Our results showed that down-regulation of the YAP expression by shRNA caused a marked reduction in cyclinD1, while the expression level of cyclinA and cyclinE were not significantly affected. Although early studies have reported that the activation of YAP increased the cyclinE transcription in Drosophila (17), our findings did not support the result in gastric cancer cells. Therefore, cyclinE was unlikely to be a transcriptional target of YAP, at least in the context of gastric cancer cells, and cyclinE transcription may be regulated by the Hippo pathway in a tissue- and region-specific manner. Therefore, our study suggested that knockdown of YAP arrested the cell cycle in the G0/G1 phase by inhibition of cyclinD1 expression.

Although Yki, the homolog of YAP, inhibited apoptosis in Drosophila (18), the role of YAP in apoptosis was controversial in mammals. Various studies have shown a proapoptotic function of YAP, which was mainly explained by the coactivation of p73 $(19,20)$. However, a discrepancy exists in the literature with regards to the role of YAP. One possible explanation is that YAP may selectively activate certain apoptotic signaling pathways, such as p73, to induce cells apoptosis in certain cells and under certain conditions. As in gastric cancer, quantification of apoptotic cells was performed by flow cytometry. The data demonstrated that the apoptosis of SGC7901 cells transfected with the YAP-shRNA vectors was significantly increased compared to that of the NC and CON groups. This suggested that YAP inhibits apoptosis in gastric cancer cells.

As a novel transcriptional coactivator, YAP is involved by binding with downstream targets (21). Therefore, an important step in future research is to identify the binding transcription factors that partner with YAP to regulate cell signaling pathways in mammals. To identify potential downstream targets of YAP, we surveyed genes whose expression were regulated by the down-regulation of YAP. Identifying these factors may provide insights into how YAP regulates the downstream signals and its role in oncogenesis. In Drosophila, activation of Yki leads to an increased transcription of Diap1, bantam, and cyclinE (17). On the other hand, reported YAP-associated transcription factors in mammals include Smad7 (22), PEBP2a (23), EBP50 (24), p53BP-2 (25), P73 (26), TEAD/TEF (27), ErbB-4 (28) and RUNX2 (29). Therefore, YAP is responsible for regulating the expression of a large number of genes and is involved in a variety of biological processes.

The most widely used proliferation factors include Ki-67 and PCNA. Ki-67 is a nuclear antigen associated with the cell cycle regulation. Results of a detailed cell cycle analysis has demonstrated that the $\mathrm{Ki}-67$ antigen was expressed in all stages with the exception of the G0 phase. Thus, Ki-67 is important for cell proliferation (30). PCNA is a $36-\mathrm{kDa}$ nuclear protein 
that acts as an auxiliary protein for DNA polymerase- $\delta$ and is an absolute requirement for DNA synthesis. It is a stable cell cycle regulated nuclear protein whose rate is directly correlated with the proliferative rate of cells (31). Survivin is a new member of the inhibitor of apoptotic protein (IAP) family. Investigators have found survivin to be overexpressed in most common tumors, but almost never in normal tissues. The overexpression of survivin is closely related to tumorigenesis and progression, and is one of the strongest apoptotic inhibitors identified (32). Early reports have shown that the expression of YAP and survivin were positively correlated and YAP may induce survivin expression in gastric cancer (33). $\alpha$-fetoprotein (AFP) is a fetal serum protein that is produced by the fetal liver and yolk sac cells, and certain fetal gastrointestinal cells (34). YAP has been found to induce AFP expression in several tumors, and clinical association analysis has revealed that YAP expression in hepatocellular carcinoma (HCC) was significantly associated with a high serum AFP level (35). Therefore, we investigated whether Ki67, PCNA, survivin and AFP were correlated with YAP expression in gastric cancer. We demonstrated that YAP down-regulation reduced the expression level of Ki67, PCNA and survivin. Therefore, we speculated that YAP induces a high expression of cell proliferation-related factors and apoptotic inhibitor factors, which may be involved in promoting gastric cancer cell proliferation and inhibiting cancer cell apoptosis. Collectively, these results suggest a regulatory role for YAP in the regulation and activation of the cell proliferation markers Ki-67 and PCNA, and the cell apoptosis marker, survivin. However, the AFP level did not have a significant difference in the YAP-shRNA group compared with the NC and CON groups. The most likely reason for this observation was that AFP was a cell-type-specific factor and was not involved in YAP regulation in gastric cancer cell line SGC7901. In brief, the genes identified in the current study suggested that YAP was an essential signaling molecule within cells, regulating a number of actions through various genes.

In conclusion, our data showed that lentivirus vectormediated RNAi significantly suppressed YAP expression in SGC7901 cells. Knockdown of YAP expression by RNAi suppressed the growth and induced apoptosis of SGC7901 cells, which may be related to the regulation of PCNA, Ki67 and survivin levels. The cell proportion of the G0/G1 phase was found to significantly increase following the silencing of YAP by down-regulating the expression of cyclinD1. These results indicate that YAP plays a significant role in gastric tumorigenesis and may therefore be a potential target for the treatment of gastric carcinoma.

\section{References}

1. Guo HQ, Guan P, Shi HL, Zhang X, Zhou BS and Yuan Y: Prospective cohort study of comprehensive prevention to gastric cancer. World J Gastroenterol 9: 432-436, 2003.

2. Dong J, Feldmann G, Huang J, et al: Elucidation of a universal size-control mechanism in Drosophila and mammals. Cell 130: $1120-1133,2007$.

3. Overholtzer M, Zhang J, Smolen GA, et al: Transforming properties of YAP, a candidate oncogene on the chromosome 11q22 amplicon. Proc Natl Acad Sci USA 103: 12405-12410, 2006.

4. Zender L, Spector MS, Xue W, et al: Identification and validation of oncogenes in liver cancer using an integrative oncogenomic approach. Cell 125: 1253-1267, 2006.
5. Steinhardt AA, Gayyed MF, Klein AP, et al: Expression of Yes-associated protein in common solid tumors. Hum Pathol 39: 1582-1589, 2008

6. Paddison PJ, Caudy AA, Bernstein E, Hannon GJ and Conklin DS: Short hairpin RNAs (shRNAs) induce sequence-specific silencing in mammalian cells. Genes Dev 16: 948-958, 2002.

7. Crombie $\mathrm{C}$ and Fraser A: Treating genetic disease through RNA interference. Lancet 365: 1288-1290, 2005.

8. Lam-Himlin DM, Daniels JA, Gayyed MF, et al: The hippo pathway in human upper gastrointestinal dysplasia and carcinoma: a novel oncogenic pathway. Int J Gastrointest Cancer 37: 103-109, 2006.

9. Qiu Z, Huang C, Sun J, et al: RNA interference-mediated signal transducers and activators of transcription 3 gene silencing inhibits invasion and metastasis of human pancreatic cancer cells. Cancer Sci 98: 1099-1106, 2007.

10. Livak KJ and Schmittgen TD: Analysis of relative gene expression data using real-time quantitative PCR and the 2(-Delta Delta C(T)) Method. Methods 25: 402-408, 2001

11. Hanahan D and Weinberg RA: The hallmarks of cancer. Cell 100: 57-70, 2000.

12. Sherr CJ and Roberts JM: CDK inhibitors: positive and negative regulators of G1-phase progression. Genes Dev 13: 1501-1512, 1999.

13. Pan D: Hippo signaling in organ size control. Genes Dev 21: 886-897, 2007.

14. Edgar BA: From cell structure to transcription: Hippo forges a new path. Cell 124: 267-273, 2006.

15. Harvey K and Tapon N: The Salvador-Warts-Hippo pathway an emerging tumour-suppressor network. Nat Rev Cancer 7: 182-191, 2007

16. Zhao B, Lei QY and Guan KL: The Hippo-YAP pathway: new connections between regulation of organ size and cancer. Curr Opin Cell Biol 20: 638-646, 2008.

17. Kango-Singh M and Singh A: Regulation of organ size: insights from the Drosophila Hippo signaling pathway. Dev Dyn 238: 1627-1637, 2009.

18. Huang J, Wu S, Barrera J, Matthews K and Pan D: The Hippo signaling pathway coordinately regulates cell proliferation and apoptosis by inactivating Yorkie, the Drosophila Homolog of YAP. Cell 122: 421-434, 2005.

19. Basu S, Totty NF, Irwin MS, Sudol M and Downward J: Akt phosphorylates the Yes-associated protein, YAP, to induce interaction with 14-3-3 and attenuation of p73-mediated apoptosis. Mol Cell 11: 11-23, 2003.

20. Strano S, Munarriz E, Rossi M, et al: Physical interaction with Yes-associated protein enhances p73 transcriptional activity. J Biol Chem 276: 15164-15173, 2001.

21. Zhang J, Ji JY, Yu M, et al: YAP-dependent induction of amphiregulin identifies a non-cell-autonomous component of the Hippo pathway. Nat Cell Biol 11: 1444-1450, 2009.

22. Ferrigno O, Lallemand F, Verrecchia F, L'Hoste S, Camonis J, Atfi A and Mauviel A Yes-associated protein (YAP65) interacts with Smad7 and potentiates its inhibitory activity against TGF-beta/ Smad signaling. Oncogene 21: 4879-4884, 2002.

23. Yagi R, Chen LF, Shigesada K, Murakami Y and Ito Y: A WW domain-containing yes-associated protein (YAP) is a novel transcriptional co-activator. Embo J 18: 2551-2562, 1999.

24. Mohler PJ, Kreda SM, Boucher RC, Sudol M, Stutts MJ and Milgram SL: Yes-associated protein 65 localizes p62(c-Yes) to the apical compartment of airway epithelia by association with EBP50. J Cell Biol 147: 879-890, 1999.

25. Espanel X and Sudol M: Yes-associated protein and p53-binding protein-2 interact through their WW and SH3 domains. J Biol Chem 276: 14514-14523, 2001.

26. Strano S, Monti O, Pediconi N, et al: The transcriptional coactivator Yes-associated protein drives p73 gene-target specificity in response to DNA Damage. Mol Cell 18: 447-459, 2005

27. Zhao B, Kim J, Ye X, Lai ZC and Guan KL: Both TEAD-binding and WW domains are required for the growth stimulation and oncogenic transformation activity of yes-associated protein. Cancer Res 69: 1089-1098, 2009.

28. Komuro A, Nagai M, Navin NE and Sudol M: WW domaincontaining protein YAP associates with ErbB-4 and acts as a co-transcriptional activator for the carboxyl-terminal fragment of ErbB-4 that translocates to the nucleus. J Biol Chem 278: 33334-33341, 2003.

29. Zaidi SK, Sullivan AJ, Medina R, et al: Tyrosine phosphorylation controls Runx2-mediated subnuclear targeting of YAP to repress transcription. Embo J 23: 790-799, 2004. 
30. Chen L, Li X, Wang GL, Wang Y, Zhu YY and Zhu J: Clinicopathological significance of overexpression of TSPAN1, Ki67 and CD34 in gastric carcinoma. Tumori 94: 531-538, 2008.

31. Schipper DL, Wagenmans MJ, Peters WH and Wagener DJ: Significance of cell proliferation measurement in gastric cancer. Eur J Cancer 34: 781-790, 1998.

32. Lehner R, Enomoto T, McGregor JA, Shroyer L, Haugen BR, Pugazhenthi U and Shroyer KR : Correlation of survivin mRNA detection with histologic diagnosis in normal endometrium and endometrial carcinoma. Acta Obstet Gynecol Scand 81: 162-167, 2002 .
33. Da CL, Xin Y, Zhao J and Luo XD: Significance and relationship between Yes-associated protein and survivin expression in gastric carcinoma and precancerous lesions. World J Gastroenterol 15: 4055-4061, 2009.

34. Lamerz R: AFP isoforms and their clinical significance (overview). Anticancer Res 17: 2927-2930, 1997.

35. Xu MZ, Yao TJ, Lee NP, et al: Yes-associated protein is an independent prognostic marker in hepatocellular carcinoma. Cancer 115: 4576-4585, 2009. 